\title{
Determination of the antiproton-to-electron mass ratio by laser spectroscopy of $\bar{p} \mathrm{He}^{+}$
}

\author{
D. Barna • A. Dax • J. Eades · K. Gomikawa • \\ R. S. Hayano • M. Hori • D. Horváth • B. Juhász • \\ N. Ono · W. Pirkl • E. Widmann • H. A. Torii
}

Published online: 29 August 2009

(C) Springer Science + Business Media B.V. 2009

\begin{abstract}
Helium is unique in the sense that about 3\% of low-energy antiprotons stopped in it survive with an average lifetime of a few microseconds, forming metastable states of the exotic antiprotonic helium atom $\left(\bar{p}-\mathrm{He}^{++}-\mathrm{e}^{-}\right)$. This lifetime is sufficient to carry out laser spectroscopy measurements of atomic transitions of this exotic atom. The antiproton-to-electron mass ratio $M_{\bar{p}} / m_{e}$ can be deduced from comparisons with three-body QED calculations. A systematic study of the energy levels of this exotic atom started soon after its discovery, continuously aiming for higher precision (for a review see Yamazaki et al., Phys Rep 366:183, (2002) and references therein). Recently, at the Antiproton Decelerator of CERN, a femtosecond optical frequency comb and continuous-wave pulse-amplified laser were used to measure 12 transition frequencies to fractional precisions of $(9-16) \times 10^{-9}$, yielding an antiproton-to-electron mass ratio of $1836.152674(5)$.
\end{abstract}

D. Barna $(\bowtie) \cdot$ A. Dax $\cdot$ K. Gomikawa $\cdot$ R. S. Hayano $\cdot$ N. Ono $\cdot$ W. Pirkl

Department of Physics, University of Tokyo, 7-3-1 Hongo, Bunkyo-ku, Tokyo 113-0033, Japan

e-mail: daniel.barna@cern.ch

J. Eades

CERN, 1211 Geneva 23, Switzerland

M. Hori

Max Planck Institut für Quantenoptik, Hans Kopfermann str. 1, 85748 Garching, Germany

D. Horváth

KFKI Research Institute for Particle and Nuclear Physics, 1525 Budapest, Hungary

B. Juhász $\cdot$ E. Widmann

Stefan Meyer Institut für Subatomare Physik, Boltzmanngasse 3, Vienna 1090, Austria

H. A. Torii

Institute of Physics, University of Tokyo, Komaba, Meguro-ku, Tokyo 153-8902, Japan 
Keywords Helium • Antiprotons • Laser spectroscopy

PACS 36.10.-k • 06.20.Dk $14.20 . \mathrm{Dh} \cdot 32.70 . \mathrm{Jz}$

\section{Introduction}

The validity of CPT symmetry can be tested in the baryon sector by comparing the properties (mass and charge, for example) of the antiproton and proton. Any small difference would disprove the validity of CPT symmetry. The energy levels of antiprotonic helium depend on these properties. High-precision laser spectroscopy of this system (backed up by 3-body QED calculations) can therefore deliver us information about the antiproton mass and charge.

The energy level diagram of $\bar{p}^{4} \mathrm{He}$ is shown in Fig. 1. The solid lines represent metastable states having $l \sim n$ ( $n$ is the principal, $l$ is the orbital angular momentum quantum number), and a typical lifetime of a few microseconds, being robust against collisions. The wavy lines indicate short-lived states, which quickly disintegrate by Auger-emission of their electron, followed by Stark-mixing with low- $l$ states and the annihilation of the antiproton on the nucleus.

The energy levels, corresponding to a given $n$ and $l$ are split into hyperfine states (not shown in this diagram). For $\bar{p}^{4} \mathrm{He}$ and $\bar{p}^{3} \mathrm{He}$ the splitting hierarchies are $\left(\mathbf{L}_{\bar{p}}+\right.$ $\left.\mathbf{S}_{e}\right)+\mathbf{S}_{\bar{p}}$ and $\left(\left(\mathbf{L}_{\bar{p}}+\mathbf{S}_{e}\right)+\mathbf{S}_{{ } \mathrm{He}_{\mathrm{e}}}\right)+\mathbf{S}_{\bar{p}}$, respectively, where $\mathbf{S}_{x}$ is the spin of the particle $x$, and $L_{\bar{p}}$ is the antiproton angular momentum.

There are two types of laser-induced transitions useful for spectroscopy. In the favoured transitions the vibrational quantum number $v=n-l-1$ does not change, whereas the unfavoured transitions change $v$ by two. For unfavoured transitions the hyperfine lines typically form two groups, with a splitting large enough to be resolved by our lasers (see for example Fig. 2b), whereas the splitting between the hyperfine lines of a favoured transition is unresolvable (Fig. 2c).

\section{Experimental procedure}

The current measurement was carried out at the Antiproton Decelerator (AD) of CERN. The 5.3-MeV antiprotons of the AD were further slowed down by a radio-frequency quadrupole decelerator to $100 \mathrm{keV}$ energies [1]. They entered a low-density $\left(T=10 \mathrm{~K}, \rho \sim 10^{18} \mathrm{~cm}^{-3}\right)$ helium gas target through a thin $(1.3 \mu \mathrm{m})$ polyethylene foil window, and stopped within a volume of about $100 \mathrm{~cm}^{3}$.

Cherenkov counters observed the charged pions produced in the annihilations of antiprotons. Their signal indicates the number of antiproton annihilations versus time. Most of the stopped antiprotons annihilate promptly, producing a burst of pions. However, about $3 \%$ of the antiprotons are captured in metastable states of antiprotonic helium, which have lifetimes of several microseconds [2]. These delayed annihilations produce a slowly-decaying component of the observed time spectrum (delayed annihilation time-spectrum, or DATS in short). In order to be able to observe this weak component, the photomultipliers reading out the Cherenkov counters were gated off during antiproton impact, and were sensitive only during the delayed component. 
Fig. 1 Energy level diagram of $\bar{p}^{4} \mathrm{He}$. The arrows represent favoured (i.e. $v=$ const) transitions

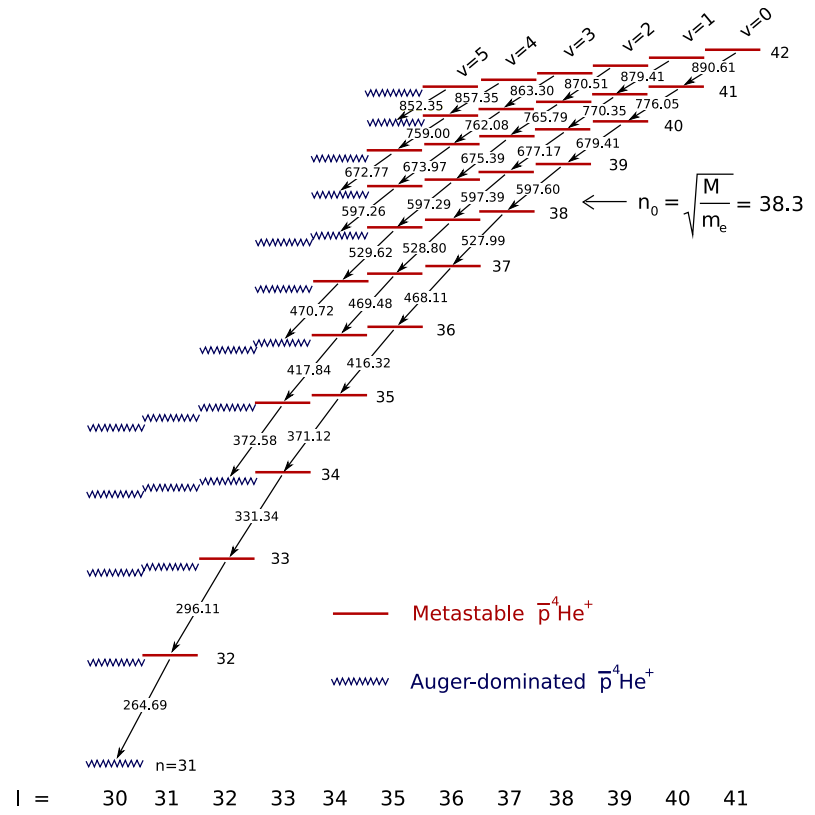

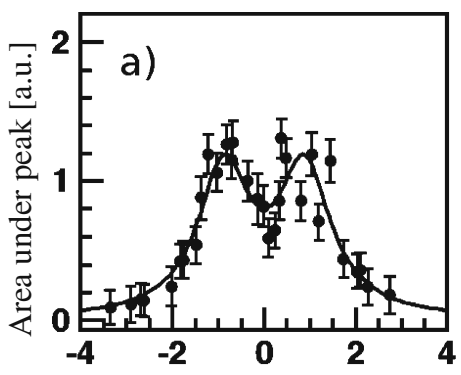
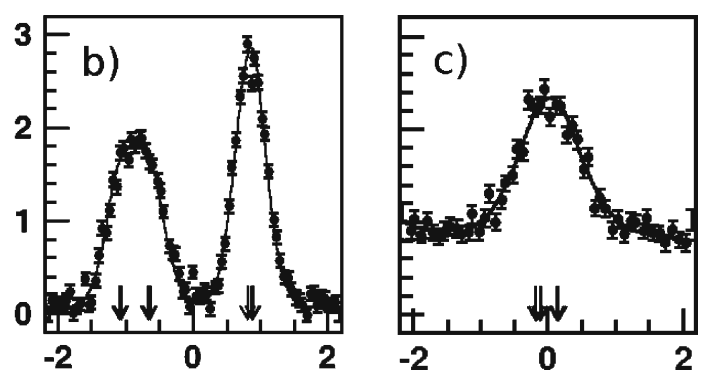

Laser frequency offset from center [GHz]

Fig. 2 Comparison of the line profiles of the $\bar{p}^{3}$ He transition $(36,34) \rightarrow(37,33)$ measured in a [1] and b [3]. c Line profile of the metastable-metastable transition $(36,34) \rightarrow(35,33)$ in $\bar{p}^{4} \mathrm{He}$. The arrows indicate the theoretical positions of the hyperfine lines

After the formation of antiprotonic helium atoms, a laser pulse irradiated the helium gas target. If the laser is tuned to an atomic transition between a metastable and short-lived state, a peak over the slowly decaying background in the timespectrum indicates the annihilation of the transferred population. The resonance profile can be measured by scanning the laser frequency through this transition, and observing the peak height versus laser frequency.

Transitions between two metastable states (for example $(n, l)=(36,34) \rightarrow(35,33)$ of $\bar{p}^{4} \mathrm{He}$ ) can be observed using 3 laser pulses. First, a tuned pulse at $t=t_{1}$, $\lambda_{1}=372.6 \mathrm{~nm}$ depletes the daughter-state $(35,33)$ of this transition into the shortlived state $(34,32)$, inducing a peak in the time-spectrum. A second laser pulse at $t=t_{2}$, scanned through $\lambda=417.8 \mathrm{~nm}$ transfers part of the population of the state 
$(36,34)$ into $(35,33)$, producing no annihilation peak in the time-spectrum, since the daughter state of this transition is also metastable. A third laser pulse at $t=t_{3}$ and $\lambda_{3}=\lambda_{1}=372.6 \mathrm{~nm}$ produces a second annihilation peak, which measures the increased population of $(35,33)$.

In order to induce the $\bar{p} \mathrm{He}$ transition, one needs laser intensities of megawatt scale. This is only available with pulsed lasers. However, reaching low fluctuations in their frequencies, and having a narrow linewidth, necessary for the precise determination of the transition line center, is very difficult. These measurements were therefore carried out using a pulse-amplified cw laser system. The cw laser seed beam was frequency-stabilized with a precision $<4 \times 10^{-10}$ against an optical frequency comb. Its intensity was pulse-amplified by a factor $10^{6}$ in three dye cells. The chirp (excursion of the frequency of the pulse from the frequency of the cw seed beam, due to a sudden change in the refractive index of the amplifying medium) was actively compensated by frequency-shifting the pulse, using an electro-optic modulator driven by an appropriate signal. The remaining chirp $\Delta v$ was measured by superimposing the pulse and a diverted, $400-\mathrm{MHz}$ frequency-shifted part of the seed $\mathrm{cw}$ laser, and observing the heterodyne beat signal. A frequency deviation of this signal from $400 \mathrm{MHz}$ indicates the chirp, which was typically $|\Delta v|<50-70 \mathrm{MHz}$. More details of the laser system can be found in [3].

\section{Analysis and results}

This measurement was part of a long and systematic study of antiprotonic helium energy levels. The precision of the results in [4] was limited by the large collisioninduced frequency-shifts $(0.5-5 \mathrm{GHz})$ of the transitions, caused by the high density of the cryogenic helium gas target (atomic density $\rho \sim 10^{21} \mathrm{~cm}^{-3}$, needed to stop the 5.3-MeV antiprotons). Extrapolation to zero density was done by measuring the density-dependence of these shifts. In our next measurement [1] the postdecelerating radio-frequency quadrupole allowed us to use a low-density cryogenic target $\left(\rho \sim 10^{17} \mathrm{~cm}^{-3}\right)$. The precision of those results was limited by the bandwidth of our pulsed laser system. The improvement in precision in our latest results is due to the new laser system. The limiting factor of these results is the Doppler-broadening of the line profiles, due to the finite temperature $T \sim 10 \mathrm{~K}$. Further improvements in precision could be reached by Doppler-free two-photon spectroscopy, the feasibility of which is currently under study.

Figure $2 \mathrm{a}$ and $\mathrm{b}$ compare the line profiles of the unfavoured $\bar{p}^{3} \mathrm{He}$ transition $(36,34) \rightarrow(37,33)$ measured in [1] and in [3]. The large hyperfine splitting was already visible with our previous laser system (Fig. 2a). The recently measured lineshape, however, shows an absolutely clear separation of these two peaks. Figure $2 \mathrm{c}$ shows the line-profile of the favoured metastable-metastable transition $(36,34) \rightarrow(35,33)$ in $\bar{p}^{4} \mathrm{He}$ measured with the new laser system. The hyperfine structure can not be resolved.

The data analysis consisted of two steps:

1) Simulation of the theoretical line profile. The hyperfine structure of $\bar{p}^{4} \mathrm{He}\left(\bar{p}^{3} \mathrm{He}\right)$ contains 4(8) intense lines, corresponding to E1 transitions involving no spinflip of any of the particles, 4(12) weaker lines involving the spin-flip of one of the constituents, and other lines even more suppressed. The theoretical 
Fig. 3 a Frequency of the $\bar{p}^{4} \mathrm{He}^{+}$transition $(37,35) \rightarrow(38,34)$ measured in the latest [3] and earlier [1,4] experiments. b

Proton-to-electron [8] and antiproton-to-electron mass ratios

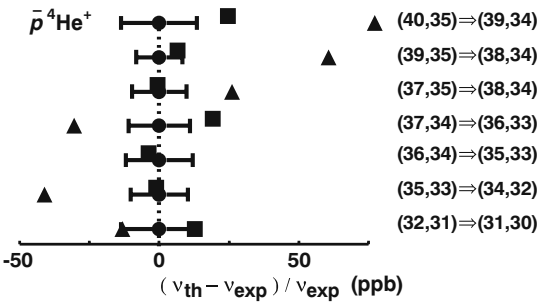

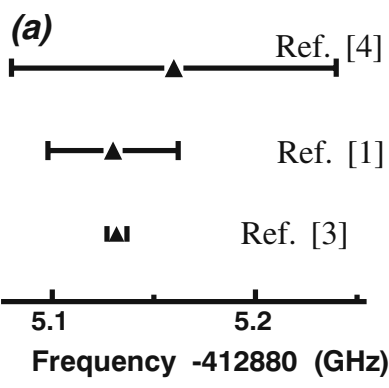

(b)

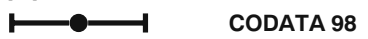

II CODATA 02

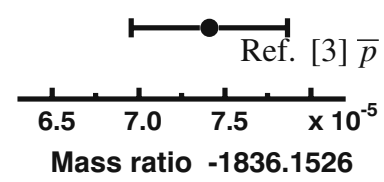

Fig. 4 Experimental $v_{\exp }$ (circles with $1 \sigma$ errorbars) vs. theoretical $v_{\text {th }}$ (triangles [6] and squares [7]) transition frequencies

line profile was obtained by numerically solving the Bloch equation, which describes the population dynamics of atomic states under the influence of laser irradiation. We took into account transitions between all hyperfine and magnetic substates, using theoretical values [5] of the above splittings (i.e. the difference from a spin-averaged, central frequency), and their dipole moments. We used the measured temporal intensity/frequency profile of the laser pulses. The Doppler-broadening effect was included by distributing the antiprotonic helium population in velocity, according to the Maxwell-Boltzmann distribution. Collisional dephasing effects were also included. Coupled transitions (sharing a common daughter/mother state, and being within the laser+chirp linewidth, i.e. being driven within the same pulse) were also simulated accordingly.

2) Fitting the spin-averaged central frequency. The simulated profile was fitted to the measured data. Since the transition frequencies have a linear collisioninduced dependence on density, final results were obtained by extrapolating to zero density using previously measured values of these density shifts [4] (typically between -14 and $1 \mathrm{MHz}$ ). The experimental error (typically 4-15 MHz) was determined as the quadratic sum of the statistical one resulting from the above fitting (3-13 MHz), and systematic ones arising from the chirp (2-4 MHz), collisional shifts (0.1-2 MHz) and the harmonic generation (1-2 MHz). The experimental results agree with previous experiments $[1,4]$ within the orderof-magnitude lower precision of the latter (Fig. 3a).

The experimental values (see ref. [3] for the numerical values) are compared to two sets of theoretical values $v_{\text {th }}[6,7]$ in Fig. 4. Values of ref. [7] (claimed precision $\sigma_{\text {th }}=1-2 \mathrm{MHz}$ ) agree within $1 \times 10^{-8}$ with the four highest-precision measurements in $\bar{p}^{4} \mathrm{He}^{+}$and $(36,34) \rightarrow(37,33)$ in $\bar{p}^{3} \mathrm{He}^{+}$. Four of its $\bar{p}^{3} \mathrm{He}^{+}$frequencies were $\sim 2 \sigma$ 
below our $v_{\exp }$ values. The results of ref. [6] scatter within 7-8 $\sigma$ of $v_{\exp }$. Unpublished results from the authors have recently moved by 3-100 $\mathrm{MHz}$ from these values. We therefore use only the ref. [7] values in the following.

The mass ratios between the proton, ${ }^{4} \mathrm{He}$ and ${ }^{3} \mathrm{He}$ nuclei, and electron $M_{p} / m_{e}=$ 1836.15267261(85), $M_{\alpha} / m_{e}=7294.2995363(32)$, and $M_{{ }^{3} \mathrm{He}} / m_{e}=5495.885269$ (11) [8] and other Committee on Data for Science and Technology (CODATA) 2002 constants were first used [7] to calculate $v_{\text {th }}$. These ratios increased by 3-6 ppb between the 1998 and 2002 compilations due to improved measurements of $m_{e}$ and $M^{3} \mathrm{He}$. We next determined the antiproton-to-electron mass ratio as the value $M_{\bar{p}} / m_{e}=$ 1836.152674(5) which gives $v_{\text {th }}\left(M_{\bar{p}} / m_{e}\right)$ values that agree best with experiment, i.e. which minimizes the sum $\sum\left[v_{\mathrm{th}}\left(M_{\bar{p}} / m_{e}\right)-v_{\exp }\right]^{2} / \sigma_{\exp }^{2}$ over the 12 measured transitions. This is consistent with the above proton value (Fig. 3b).

We can also set a new upper limit $\delta_{\bar{p}}$ on any CPT-violating difference between the antiproton and proton charges $\left(Q_{\bar{p}}, Q_{p}\right)$ and masses using the values of $v_{\text {th }}$ and $v_{\exp }$ and the equation [2] $\delta_{\bar{p}}=\left(Q_{p}+Q_{\bar{p}}\right) / Q_{p} \sim\left(M_{p}-M_{\bar{p}}\right) / M_{p}=\kappa\left(v_{\text {th }}-v_{\exp }\right) / v_{\exp }$. Values for $\kappa=2.5-6.1$ were estimated [6] by increasing $Q_{\bar{p}}$ and $M_{\bar{p}}$ by 1 ppm under the constraint on $Q_{\bar{p}} / M_{\bar{p}}$ against $Q_{p} / M_{p}$ of $<9 \times 10^{-11}$ [9] and noting the change in $v_{\text {th }}$. The average value of $\delta_{\bar{p}}$ over 12 transitions was $(-3 \pm 14) \times 10^{-10}$, which implies that the antiproton charge and mass agree with those of the proton to a precision of $2 \times 10^{-9}$ at a confidence level of $90 \%$. The precisions of these determinations were partially limited by the differences between $v_{\text {th }}$ and $v_{\exp }$ in $\bar{p}^{3} \mathrm{He}^{+}$.

Acknowledgements We are deeply indebted to D. Bakalov, K.S.E. Eikema, M. Fischer, R. Holzwarth, Y. Kino, V.I. Korobov, K. Jungmann, and Th. Udem. This work was supported by the Global COE Program "Physical Sciences Frontier" of MEXT, Japan, the Grant-in-Aid for Specially Promoted Research (20002003) of MEXT, Japan, the Mitsubishi Foundation, the EURYI award of the European Science Foundation and the Deutsche Forschungsgemeinschaft, the Munich Advanced Photonics (MAP) cluster, the Max-Planck-Gesellschaft, and the Hungarian Science Foundation and the Hungarian National Bureau of Science and Development (Contracts OTKA NK67974, K72172, H07C-74153 and JAP-21/2006).

\section{References}

1. Hori, M., et al.: Phys. Rev. Lett. 91, 123401 (2003)

2. Yamazaki, T., Morita, N., Hayano, R. S., Widmann, E., Eades, J.: Phys. Rep. 366, 183 (2002)

3. Hori, M., et al.: Phys. Rev. Lett. 96, 243401 (2006)

4. Hori, M., et al.: Phys. Rev. Lett. 87, 093401 (2001)

5. Korobov, V.I.: Phys. Rev. A 73, 022509 (2006)

6. Kino, Y., Kudo, H., Kamimura, M.: Mod. Phys. Lett. A 18, 388 (2003) (private communication)

7. Korobov, V.I.: In: Hirtl, A., Marton, J., Widmann, E., Zmeskal, J. (ed.) Proceedings of the EXA05 International Conference on Exotic Atoms and Related Topics. Austrian Academy of Sciences Press, Vienna (2005)

8. Mohr, P.J., Taylor, B.N.: Rev. Mod. Phys. 72, 351 (2000); 77, 1 (2005)

9. Gabrielse, G., et al.: Phys. Rev. Lett. 82, 3198 (1999) 